\title{
Poaceae endémicas del Perú
}

\author{
${ }^{1}$ Museo de Historia Natu- \\ ral, Av. Arenales 1256, \\ Aptdo 14-0434, Lima 14, \\ Perú. \\ marycano 11@yahoo.com \\ ${ }^{2}$ Herbario del Departamen- \\ to de Biología (MOL), Fa- \\ cultad de Ciencias, Univer- \\ sidad Nacional Agraria La \\ Molina, Aptdo. 456, Lima, \\ Perú. \\ grmn@lycos.com \\ ${ }^{3}$ Rancho Santa Ana Botanic \\ Garden, 1500 N College \\ Ave., Claremont, CA91711, \\ EE.UU. \\ refulio@mailcity.com \\ ${ }^{4}$ Universidad Nacional de \\ Cajamarca, Apartado 55, \\ Cajamarca, Perú. \\ svisidoro@yahoo.com
}

\author{
María Isabel La Torre ${ }^{1}$, José Alegría ${ }^{2}$, Nancy Refulio ${ }^{3}$ e Isidoro Sánchez ${ }^{4}$
}

\section{Resumen}

La familia Poaceae es reconocida en el Perú por presentar alrededor de 157 géneros y 750 especies (Brako \& Zarucchi, 1993; Ulloa Ulloa et al., 2004), principalmente hierbas. En este trabajo reconocemos 81 endemismos en 19 géneros. Estos taxones endémicos se encuentran en prácticamente todas las regiones ecológicas, aunque la mayor concentración se encuentra en la Puna Húmeda y Seca, Alto Andina y Mesoandina, desde el nivel del mar hasta los $5500 \mathrm{~m}$ de altitud. Veinticinco taxones se encuentran representados dentro de áreas naturales protegidas.

Palabras claves: Poaceae, Perú, endemismo, plantas endémicas.

\section{Abstract}

The Poaceae are represented in Peru by 157 genera and 750 species (Brako \& Zarucchi, 1993; Ulloa Ulloa et al., 2004), mostly herbs. Here we recognize 81 endemic species in 19 genera. Peru's endemic grasses have been found in practically all recognized ecological regions, although the majority is found in the Dry and Humid Puna, High Andean and Mesoandean regions, from sea level to $5500 \mathrm{~m}$ elevation. Twenty-five endemics have been reported to occur in Peru's protected areas.

Keywords: Poaceae, Peru, endemism, endemic plants.

\section{Aristida chiclayensis Tovar}

$$
\text { EN, Bla }
$$

Publicación: Publ. Mus. Hist. Nat. «Javier Prado», Ser. B, Bot. 32: 11. 1984.

Colección tipo: R. Ferreyra 6047

Herbarios: US; MOL!

Nombre común: Barba de chivato.

Registro departamental: CA, LA, LL, PI.

Regiones Ecológicas: DCT, D ST; $150-800 \mathrm{~m}$.

SINANPE: Sin registro.

Herbarios peruanos: HAO (3), MOL (isotipo).

Observaciones: Especie herbácea anual, conocida de la vegetación costera estacional, de poblaciones naturalmente fragmentadas, soamente del norte del país. Probablemente su presencia está influenciada por eventos El Niño. Amenazas a algunas de las poblaciones están asociadas a la expansión urbana.

\section{Aulonemia humillima (Pilg.) McClure}

\section{NT}

Publicación: Smithsonian Contr. Bot. 9: 58. 1973. Colección tipo: E.H.G. Ule 6598

Herbarios: B, US.

Nombre común: D esconocido.

Registro departamental: AM, JU, PA, SM.

Regiones Ecológicas: BMHM, BMHP; 910- 2000 m. SINAN PE: ACRCE

Herbarios peruanos: AMAZ (2), USM (2).

Observaciones: Este bambú se conoce del norte y del centro del país, de lugares impactados por deforestación natural 0 antropogénica. Se estima que el área de presencia alcanza casi los $1000 \mathrm{~km}^{2}$. Localmente forma poblaciones densas. La localidad tipo fue considerada originalmente en el departamento de Loreto, pero corresponde hoy a San Martín. Esta localidad, Cerro La Escalera, alberga otros endemismos y ha sido considerada en 2005, Área de Conservación Regional Cordillera Escalera.
3. Bromus striatus Hitchc.

\section{VU, Blab(iii)c(ii,iv)}

Publicación: Contr. U.S. Natl. Herb. 24(8): 316. 1927.

Colección tipo: A. Hitchcock 22386

Hemarios: NY, US.

Nombre común: Soclla.

Registro departamental: AR, AY, LI, LL, MO.

Regiones Ecológicas: DST; 250- $700 \mathrm{~m}$.

SINANPE: RNL

Herbarios penuanos: HUT (10), MOL (2), USM (14).

Observaciones: Gramínea anual conocida de varias poblaciones dispersas, en ambientes naturalmente fragmentados, en la costa peruana. Las localidades más distantes se hallan separadas por más de $800 \mathrm{~km}$. Todas las colecciones fueron hechas en las formaciones de lomas costaneras. Su rango geográfico es pequeño, menor de $5000 \mathrm{~km}^{2}$. Sus poblaciones están sujetas a perturbación o destrucción de su hábitat debido a la acción humana.

\section{Calamagrostis cuzcoensis Tovar}

\section{CR, Blab(iii)}

Publicación: Publ. Mus. Hist. Nat. «Javier Prado», Ser. B, Bot. 33: 11. 1985.

Colección tipo: O. Tovar \& S. Rivas-Martinez 8905

Hemarios: MAF, MO, US; USM!.

Nombre común: D esconocido.

Registro departamental: CU.

Regiones Ecológicas: AA; $4230 \mathrm{~m}$.

SINAN PE: Sin registro.

Herbarios penuanos: USM (holotipo).

Observaciones: Especie herbácea perenne, conocida de una localidad, en el sur del país. El hábitat de esta población puede estar afectado por actividades antropogénicas, como la quemay el pastoreo intensivo. Especie conocida sólo por el tipo, recolectado en 1981, en el Abra Málaga, en la carretera Cuzco-Q uillabamba. Estalocalidad se ubica a unos $10 \mathrm{~km}$ del Santuario Histórico Machu Picchu; es probable su presencia en aquella área protegida.

Nota del Editor: En la versión on line de este artículo han sido omitidos los mapas del Perú que ilustraban el Registro departamental. Para ubicar las abreviaturas de los departamentos vea al final del artículo. 


\section{Calamagnostis macbridei Tovar}

LC

Publicación: Mem. Mus. Hist. Nat. «Javier Prado» 11: 62. 1960.

Colección tipo: J.F. Macbride \& W.

Featherstone 998

Herbarios: MO, US.

Nombre común: D esconocido.

Registro departamental: AN, AY, CA, JU, PA, PU.

Regiones Ecológicas: MA, PSH, PD, AA; 3260- $4600 \mathrm{~m}$.

SINANPE: PNH

Herbarios peruanos: USM (11)

Observaciones: Esta gramínea perenne se conoce de varias localidades, en el centro y sur del país. Esta es una especie bastante frecuente en ecosistemas andinos y altoandinos. Las poblaciones son extensas y numerosas, y están presentes en un amplio rango altitudinal. Recolectas recientes y la amplitud de su distribución hacen suponer que el estado de conservación de sus poblaciones es de preocupación menor. Existe la posibilidad de su presencia en la Reserva Nacional de Junín, ya que la localidad de la colección tipo, Huarón, se encuentra a unos 20 km de esta área.

\section{Calamagrostis pungens Tovar}

\author{
NT \\ Publicación: Mem. Mus. Hist. Nat. «Javier \\ Prado» 11: 61. 1960 \\ Colección tipo: E. Salaverry 106 \\ Herbarios: MO, US. \\ Nombre común: D esconocido. \\ Registro departamental: AN, HV, JU, LL. \\ Regiones Ecológicas: $\mathrm{PSH}, \mathrm{PAR}, \mathrm{AA}$; \\ 3550- $4560 \mathrm{~m}$. \\ SINANPE: Sin registro. \\ Herbarios penuanos: CPUN (1), USM (4).
}

Observaciones: Esta especie se conoce del norte y centro del país, dondees localmente frecuente. Ha sido recolectada consecutivamente durantelosúltimos 50 años. Las probables amenazas a sus poblaciones están asociadas a la remoción de suelos, por la actividad minera o la quema de pajonales para el pastoreo.

\section{Calamagrostis rauhii Tovar}

\section{EN, Blab(iii)}

Publicación: Mem. Mus. Hist. Nat. «Javier Prado» 11: 78. 1960.

Colección tipo: W. Rauh \& G. Hirsch P1724

Herbarios: MO, US.

Nombre común: D esconocido.

Registro departamental: JU, LL.

Regiones Ecológicas: AA; 4200-4500 m.

SINANPE: Sin registro.

Herbarios peruanos: USM (1).

Observaciones: Esta especie se conoce de dos localidades, en el centro y norte del país, al parecer, no ha vuelto a ser recolectada desde 1982. Probablemente con una mayor exploración y recolecta de las partes altoandinas se contribuya al conocimiento de esta especie. Amenazas probables a sus poblaciones estén asociadas ciertamente a algunas prácticas agrícolas y la minería.
8. Chusquea aspera L.G. Clark

EN, B1a; D2

Publicación: Iowa State J. Res. 61(1): 113, f. 4 f-i. 1986.

Colección tipo: J. Schunke V. 10178

Hembarios: F, ISC, K, MO, SI, US, VEN.

Nombre común: Marona sin hueco.

Registro departamental: AM, CA, HU.

Regiones Ecológicas: BMHM; 1600$3000 \mathrm{~m}$.

SINANPE: PNCA

Hembarios peruanos: USM (1).

Observaciones: Este bambú trepador se conoce de varias localidades, en ambas vertientes andinas, pero localmente escasa. Según Tovar (1993) podría estar presente en otros bosques montanos. Los ambientes, de donde se conoce esta especie, están sujetos a deforestación. Las localidades más distantes entre ellas se hallan cerca de $600 \mathrm{~km}$ aparte.

\section{Chusquea barbata L.G. Clark}

\section{CR, Bla}

Publicación: Novon 3(3): 232, 235, f. 2. 1993.

Colección tipo: A.H. Gentry et al. 42007

Hembarios: ISC, MO, US; AMAZ!, USM!.

Nombre común: D esconocido.

Registro departamental: PA.

Regiones Ecológicas: $\mathrm{BMHP}, \mathrm{BHA}$; 400- $700 \mathrm{~m}$.

SINAN PE: BPSMSC

Herbarios peruanos: AMAZ (isotipo), USM (isotipo).

Observaciones: Bambú conocido del único ejemplar recolectado en Pasco, en el bosque de protección San Matías-San Carlos cerca al Parque Nacional Yanachaga-Chemillén. Probablemente, con exploraciones y herborizaciones adicionales, en las zonas premontanas y bajas de Pasco, pueda contribuirse a adicionar información sobre las poblaciones de esta especie.

\section{Chusquea decolorata Munno ex L.G. Clark}

\section{DD}

Publicación: J. Amer. Bamboo Soc. 18(1): 25-28, 24, f. 1-2. 2004.

Colección tipo: M. Pavón s.n.

Herbarios: G, K, P.

Nombre común: D esconocido.

Registro departamental: Sin datos.

Regiones Ecológicas: Sin datos; altitud desconocida.

SINANPE: Sin registro.

Herbarios peruanos: Ninguno.

Observaciones: No se conoce la localidad precisa, ni el año, en que fue recolectado el tipo. Se le asigna la categoría Datos Insuficientes. Se requiere aclarar su estado taxonómico y distribución; investigaciones futuras podrían mostrar muy bien que una clasificación de amenazado sería apropiada. 


\section{Chusquea huantensis Pilg.}

\section{VU, B 1a; D2}

Publicación: Bot. Jahrb. Syst. 56(Beibl. 123): 29. 1920.

Colección tipo: A. Weberbauer 5581

Herbarios: MO, US.

Nombre común: D esconocido.

Registro departamental: AY, CU, HV.

Regiones Ecológicas: BMHM; 2900$3000 \mathrm{~m}$.

SINANPE: Sin registro.

Hemarios peruanos: USM (1).

Observaciones: Bambú conocido solamente de los bosques montanos de tres localidades en el centro y sur del país. Aparentemente no ha vuelto a ser recolectada desde 1954.

\section{Chusquea inamoena Pilg.}

\section{CR, B 1ab(iii)}

Publicación: Repert. Spec. Nov. Regni Veg. 1(10): 150. 1905.

Colección tipo: A. Weberbauer 2295

Herbarios: MO, US.

Nombre común: D esconocido.

Registro departamental: JU.

Regiones Ecológicas: BMHM; $2700 \mathrm{~m}$.

SINAN PE: Sin registro.

Herbarios peruanos: Ninguno.

Observaciones: Bambú conocido solamente de una localidad, en el centro del país. Tovar (1993) la incluyó en la sinonimia de Chusquea serulata, pero Clark (2000) considera a este bambú como una especie distinta, criterio con el cual concordamos. La zona original, en Huacapistana, está fuertemente afectada por la deforestación.

\section{Chusquea picta Pilg.}

\section{NT}

Publicación: Repert. Spec. Nov. Regni Veg. 1(10): 151. 1905.

Colección tipo: H. Ruíz s.n.

Herbarios: B, MO, US.

Nombre común: D esconocido.

Registro departamental: HU, PA, SM.

Regiones Ecológicas: BMHM; 2550$3350 \mathrm{~m}$.

SINANPE: PNRA, PNYC

Herbarios peruanos: HAO (1), HUT (1), USM (2)

Observaciones: Bambú conocido de cuatro localidades en el norte y centro de la vertiente oriental. Las recolectas recientes de esta especie provienen de áreas protegidas y poco se sabe de la situación de esta especie fuera de ellas.

\section{Chusquea pubispicula Pilg.}

\section{VU, B 1ab(iii), D2}

Publicación: Repert. Spec. Nov. Regni Veg. 1(10): 148. 1905.

Colección tipo: A. Weberbauer 688

Herbarios: US.

Nombre común: Sinua.

Registro departamental: CU, HV, PU. Regiones Ecológicas: BMHM; 2500$3100 \mathrm{~m}$.

SINANPE: SHMP

Herbarios peruanos: USM (4).
Observaciones: Bambú que forma comunidades densas y que morfológicamente asemeja a Chusquea scandens. Se conoce de cuatro poblaciones en el sur del país. Tovar (1993) comentó la posibilidad que podría encontrarse en Bolivia. Se conoce del Santuario Histórico Machu Picchu, donde está sujeta a tala. Se le asigna la categoría Vulnerable por presentar un rango geográfico menor de $20000 \mathrm{~km}^{2}$.

\section{Chusquea smithii L.G. Clark}

\section{CR, Bla}

Publicación: Iowa State J. Res. 61(1): 107, f. 2 g-i. 1986.

Colección tipo: D.N. Smith 7730

Hemarios: ISC, K, MO, US; AMAZ!.

Nombre común: D esconocido.

Registro departamental: PA.

Regiones Ecológicas: $\mathrm{BPM}, \mathrm{BMHM}$; 3350- $3620 \mathrm{~m}$

SINAN PE: PNYC

Herbarios penuanos: AMAZ (isotipo).

Observaciones: Bambú conocido solamente de una población, en un área protegida. No ha vuelto a ser recolectada desde 1984, probablemente por lo poco herborizada que es lazona. No se conoce de amenazas a sus poblaciones.

\section{Chusquea straminea Pilg.}

\section{CR, Blab(iii)}

Publicación: Repert. Spec. Nov. Regni Veg. 1(10): 147. 1905.

Colección tipo: A. Weberbauer 4408

Hemarios: B, US; MOL!.

Nombre común: Desconocido.

Registro departamental: AM.

Regiones Ecológicas: BMHM; 2300$2600 \mathrm{~m}$

SINAN PE: Sin registro.

Hemarios penuanos: MOL (isotipo).

Observaciones: Bambú conocido de una localidad en el norte del país, en la vertiente oriental, en la cuenca del Utcubamba. Tovar (1993) la consideró en la sinonimia de Chusquea scandens, pero Clark (2000) la segregó. El rango de distribución no supera los 100 km². Una recolecta adicional de Cajamarca (Diaz \& Osores 2978 MO) probablemente represente este taxón. La compleja taxonomía de Chusquea y la falta de exploración, pues no ha vuelto a ser registrada desde 1904, contribuyen a su poco conocimiento.

\section{Chusquea tarmensis Pilg.}

\section{VU, Bla}

Publicación: Repert. Spec. Nov. Regni Veg. 1(10): 151. 1905.

Colección tipo: A. Weberbauer 2129

Hemarios: B, US.

Nombre común: D esconocido.

Registro departamental: CA, CU, JU, PA. Regiones Ecológicas: BMHM; 2100$2600 \mathrm{~m}$.

SINANPE: Sin registro.

Henbarios penuanos: USM (3). 
Observaciones: Este bambú endémico es el de mayor rango geográfico, conociéndose de Cajamarca a Cusco. Crece asociado a bosques que reciben alta nubosidad y presentan abundantes musgos y helechos. Las recolectas más recientes provienen de los inicios de la década de 1980. No se conoce el estatus de las poblaciones, pero por su rango altitudinal podrían estar afectadas por la deforestación. Se considera esta especie Vulnerable esperando que promueva el interés por una evaluación detallada de la misma.

\section{Dissanthelium amplivaginatum Tovar}

$$
\text { VU, Bla }
$$

Publicación: Publ. Mus. Hist. Nat. «Javier Prado», Ser. B, Bot. 33: 7. 1985.

Colección tipo: 0 . Tovar \& S. RivasMartinez 9777

Henbarios: MAF, US; USM!

Nombre común: D esconocido.

Registro departamental: AN.

Regiones Ecológicas: AA; 4700-4870 m.

SINAN PE: PNH

Hembarios penuanos: USM (holotipo+2).

Observaciones: Esta gramínea se conoce solamente de unas pocas localidades, en la Cordillera Blanca, donde es localmente abundante. El hábitat de esta especie está en buen estado de conservación, por localizarse a altitudes cercanas a los $5000 \mathrm{~m}$, generalmente poco accesibles para el ganado.

\section{Dissanthelium brevifolium Swallen \& Tovar}

\section{VU, Blab(iii)}

Publicación: Phytologia 11(6): 375-376. 1965.

Colección tipo: J.F. Macbride \& W. Featherstone 933

Herbarios: F, US.

Nombre común: D esconocido.

Registro departamental: JU, PA.

Regiones Ecológicas: PSH; 4100-4115 m.

SINAN PE: Sin registro.

Hembarios peruanos: Ninguno.

Observaciones: Hierba perenne conocida de cuatro localidades, en el centro del país. Al igual que otras especies de la puna, probables amenazas a sus poblaciones están asociadas al pastoreo intensivo e incendios intencionales. No se hallaron recolectas de menos de diez años atrás.

\section{Dissanthelium giganteum Tovar}

\section{VU, Bla}

Publicación: Publ. Mus. Hist. Nat. «avier Prado», Ser. B, Bot. 33: 8. 1985.

Colección tipo: O. Tovar \& S. RivasMartinez 9831

Henbarios: MAF, US; USM!.

Nombre común: D esconocido.

Registro departamental: AN, JU.

Regiones Ecológicas: AA; 4500-4870 m.

SINANPE: PNH

Herbarios penuanos: USM (holotipo+2).

Observaciones: Esta especie se conoce del centro del país y se caracteriza por el tamaño grande de las macollas. Sin embargo, solamente tres poblaciones se conocen de esta especie. En la Cordillera Blanca crece junto con otra endémica, D issanthelium amplivaginatum.

\section{D issanthelium longifolium Tovar}

\section{VU, Bla}

Publicación: Publ. Mus. Hist. Nat. «avier Prado», Ser. B, Bot. 33: 9. 1985.

Colección tipo: O. Tovar et al. 9884

Herbarios: MAF, US; USM!.

Nombre común: D esconocido.

Registro departamental: AN, HU.

Regiones Ecológicas: PSH; 4000-4100 m.

SINANPE: Sin registro.

Herbarios peruanos: USM (holotipo+1).

Observaciones: Esta especie perenne se conoce solamente de dos localidades, en el centro del país. Las poblaciones conocidas son dispersas y probablemente esto contribuya a su rareza.

\section{Dissanthelium pygmaeum Swallen \& Tovar}

$$
\text { EN, B1ab(iii) }
$$

Publicación: Phytologia 11(6): 367-368. 1965.

Colección tipo: 0 . Tovar 2545

Herbarios: US; USM!.

Nombre común: D esconocido.

Registro departamental: HV.

Regiones Ecológicas: AA; 4500-4800 m.

SINANPE: Sin registro.

Herbarios peruanos: USM (holotipo+1).

Observaciones: Esta especie perenne se conoce solamente de dos localidades,en la cuenca del río Mantaro. La escasez de registro botánico es el tema dominante para la flora andina; se espera que con la exploración y estudio de esa parte del país, se pueda aclarar el estatus de conservación de esta especie. No se conoce de recolectas adicionales desde 1961.

\section{Dissanthelium rauhii Swallen \& Tovar}

\section{VU, Bla}

Publicación: Phytologia 11(6): 376. 1965. Colección tipo: W. Rauh \& G. Hirsch P-1418

Henbarios: HEID, MO, US.

Nombre común: D esconocido.

Registro departamental: CU.

Regiones Ecológicas: MA, PSH; 3200$3700 \mathrm{~m}$.

SINANPE: PNM

Herbarios peruanos: USM (6).

Observaciones: Esta gramínea perenne se conoce solamente del sur del país, de cinco localidades en tres de las provincias de Cusco. Es una especie localmente frecuente y hay recolectas recientes en un área protegida. Sin embargo, las poblaciones en el Parque Nacional Manu están sujetas a incendios intencionales que sobrepasan los límites del parque.

\section{Dissanthelium semitectum Swallen \& Tovar}

DD

Publicación: Phytologia 11(6): 370. 1965. Colección tipo: J.F. Macbride \& W. Featherstone 1155

Herbarios: US.

Nombre común: D esconocido.

Registro departamental: PA.

Regiones Ecológicas: AA; 4200 m.

SINANPE: Sin registro.

Henbarios peruanos: Ninguno. 
Observaciones: Esta especie se conoce solamente de una localidad, ubicada en Pasco, en la que no ha vuelto a ser recolectada desde 1922.

\section{Eragrostis ancashensis P.M. Peterson, Refulio \& Tovar}

\section{DD}

Publicación: Sida 19(1): 66, f. 1. 2000. Colección tipo: P.M. Peterson \& N. Refulio 13793

Hemanios: K, MO, NY, RSA, TAES, UC, US, WISC; USM!.

Nombre común: D esconocido.

Registro departamental: AN.

Regiones Ecológicas: MA, PSH; 2200$4150 \mathrm{~m}$.

SINAN PE: Sin registro.

Herbarios peruanos: USM (holotipo+5).

Observaciones: Esta especie se conoce sólo de las vertientes occidentales, de la Cordillera Blanca. Es considerada por Alegría \& Granda (2001) como coespecífica con E ragrostis pilgeri. Esta incertidumbre taxonómica requiere aclararse.

\section{Eragrostis magna Hitchc.}

\section{CR, Bla}

Publicación: Contr. U.S. Natl. Herb. 24(8): 341. 1927.

Colección tipo: J.F. Macbride 4069

Herbarios: F, US

Nombre común: D esconocido.

Registro departamental: HU.

Regiones Ecológicas: BMHM; 2000 m.

SINANPE: Sin registro.

Herbarios peruanos: Ninguno.

Observaciones: Hierba perenne, la cual no se le ha vuelto a encontrar desde que J.F. Macbride recolectó el tipo en 1923. La localidad original, en la cuenca alta del Huallaga, ha sido escasamente herborizada.

\section{Eragrostis pilgeri Fedde}

\section{VU, B lab(iii)}

Publicación: Just's Bot. Jahresber 3: 18, t. 34. 1908.

Colección tipo: A. Weberbauer 3114

Hembarios: BAA, US; MOL!.

Nombre común: D esconocido.

Registro departamental: AN, CA.

Regiones Ecológicas: MDE, MA, PSH; 2200- $4150 \mathrm{~m}$.

SINAN PE: PNH

Herbarios peruanos: CPUN (6), MOL (lectotipo+2), USM (7).

Observaciones: Esta gramínea perenne se conoce de varias localidades, en un área de presencia menor a los $20000 \mathrm{~km}^{2}$. Ampliamente distribuida en el departamento de Áncash, especialmente en la Cordillera Negra. Sólo se ha registrado una población en la Cordillera Blanca (Parque Nacional Huascarán).

\section{Festuca cajamarcae Pilg.}

EN, Blab(iii)

Publicación: Bot. Jahrb. Syst. 37: 513514. 1906.

Colección tipo: A. Weberbauer 3974

Herbarios: B, BAA, US; MOL!

Nombre común: Desconocido.

Registro departamental: CA, LL.

Regiones Ecológicas: PAR; 3200-4100 $\mathrm{m}$.

SINANPE: Sin registro.

Herbarios peruanos: CPUN (6), MOL (isotipo), USM (1).

Observaciones: Planta cespitosa y perenne conocida de las jalcas del norte del país, donde es localmente frecuente. Esta especie ha sido considerada como restringida a Cajamarca (Tovar, 1993; D arbyshire et al., 2003), pero recolectas recientes amplían el área de distribución hacia el sur. Amenazas a las poblaciones de esta especie y con ella de otras plantas de la jalca provienen de los incendios intencionales con propósitos de pastoreo. Por su área de presencia menor a los $5000 \mathrm{~km}^{2}$ se la reconoce como amenazada.

\section{Festuca carazana Pilg.}

LC

Publicación: Bot. Jahrb. Syst. 37: 511512. 1906.

Colección tipo: A. Weberbauer 3106

Herbarios: B, BAA, MO, US; MOL!.

Nombre común: Desconocido.

Registro departamental: AN, CA, HU, LL, SM.

Regiones Ecológicas: PSH, PAR, AA; 3500- $4400 \mathrm{~m}$.

SINANPE: PNH

Herbarios penuanos: MOL (isotipo), HUT (1), USM (9).

Observaciones: G ramínea que forma macollas grandes y se conoce de la zona norte del país y de las vertientes orientales del centro. Está bien representada en los herbarios, representando varias poblaciones. Probablemente las amenazas sean similares a otras plantas de la puna, pero por su área de distribución y representación se la reconoce como de preocupación menor.

\section{Festuca densiflora Tovar}

\section{VU, B1ab(iii), D2}

Publicación: Mem. Mus. Hist. Nat. «Javier Prado» 16: 71, t.16B. 1972.

Colección tipo: W. Rauh \& G. Hirsch P1223

Herbarios: US.

Nombre común: D esconocido.

Registro departamental: AN, CU.

Regiones Ecológicas: AA; 4500-4720 m.

SINAN PE: Sin registro.

Herbarios penuanos: USM (1).

Observaciones: Esta gramínea perenne que se conoce de dos localidades, distantes entre ellas más de $500 \mathrm{~km}$. En general, la escasez de colecciones de esta especie refleja la escasa recolecta botánica de las zonas por encima de los $4000 \mathrm{~m}$ de altitud. Por la ausencia de protección oficial y por la presencia fragmentada de sus poblaciones se la considera amenazada. 


\section{Festuca dentiflora E.B. Alexeev ex Stanèíck \& P.M. Peterson}

CR, Blab(iii)

Publicación: Sida 20(3): 1016, f. 1. 2003. Colección tipo: C. Wilkes, Exped. Expl. US. 5

Herbarios: US.

Nombre común: D esconocido.

Registro departamental: $\mathrm{HU}$.

Regiones Ecológicas: MA; altitud desconocida.

SINANPE: Sin registro.

Herbarios peruanos: Ninguno.

Observaciones: Esta especie se conoce solamente de la localidad original,en el centro del país. No ha vuelto a ser recolectada desde el siglo XIX. No se cuenta con la información acerca del estado de las poblaciones y de los hábitats.

\section{Festuca glabrata Tovar}

\section{VU, Bla; D2}

Publicación: Mem. Mus. Hist. Nat. «Javier Prado» 16: 30, t. 6A. 1972.

Colección tipo: A. Hitchcock 22453

Herbarios: US.

Nombre común: D esconocido.

Registro departamental: CU, HV.

Regiones Ecológicas: PSH; 3400-3600

m.

SINANPE: PNM

Herbarios peruanos: USM (2).

Observaciones: Planta perenne conocida del centro-sur del país, donde ha sido escasamente recolectada. El ejemplar de herbario más reciente proviene de 1990. Amenazas potenciales a esta especie provienen del pastoreo y de los incendios asociados.

\section{Festuca glyceniantha Pilg.}

\section{LC}

Publicación: Bot. Jahrb. Syst. 37: 516517. 1906.

Colección tipo: A. Weberbauer 3275

Herbarios: B, US; MOL!

Nombre común: D esconocido.

Registro departamental: AN, CA, LL, PA. Regiones Ecológicas: PSH, PAR; 3340$4200 \mathrm{~m}$.

SINAN PE: PNH

Herbarios peruanos: MOL (isotipo+1), USM (4).

Observaciones: Planta perenne conocida de varias localidades, en el norte y centro del país. Esta especie fue recolectada originalmente de una localidad hoy en el Parque Nacional Huascarán. Amenazas potenciales probablemente estén asociadas a la quema intencional y pastoreo intensivo. Falta sin embargo información sobre la historia natural y características de las poblaciones de esta especie.

\section{Festuca horridula Pilg.}

LC

Publicación: Bot. Jahrb. Syst. 37: 514515. 1906

Colección tipo: A. Weberbauer 2586

Herbarios: B, BAA, US; $\underline{\text { MOL! }}$.

Nombre común: Chilín, maugalaria.

Registro departamental: AN, AY, CA, HV, JU, LL, LI, PA.

Regiones Ecológicas: MA, PSH, PAR; $2600-4000 \mathrm{~m}$.

SINANPE: PNH

Herbarios penuanos: HAO (1), HUT (2), MOL (isotipo), USM (19).

Observaciones: Esta especie es una de las gramíneas endémicas de mayor rango de distribución geográfica y altitudinal. Ha sido recolectada en varias oportunidades durante el siglo XX. Cuenta con poblaciones en un área protegida, además las que se conocen fuera de esta área parecen no tener amenazas mayores, tal vez por encontrarse en ambientes rocosos.

\section{Festuca huamachucensis Infantes}

\section{NT}

Publicación: Revista Ci. (Lima) 54: 107. 1952.

Colección tipo: J. Infantes 3738

Hemarios: US.

Nombre común: D esconocido.

Registro departamental: AN, AY, CA, HU, JU, LL.

Regiones Ecológicas: MA, PSH, PAR, AA; 2700-4750 m.

SINANPE: PNH, RNJ

Herbarios penuanos: CPUN (6), HAO (2), USM (8).

Observaciones: Esta gramínea se conoce, al igual que Festuca horridula, de varias localidades en el norte y centro del país en un amplio rango ecológico. Se sabe que la localidad original está fuertemente afectada por los incendios intencionales asociados al pastoreo.

\section{Festuca lanatifolia Tovar}

$$
\text { VU, B1a; D2 }
$$

Publicación: Mem. Mus. Hist. Nat. «Javier Prado» 16: 22, t.4A. 1972.

Colección tipo: E. Asplund 11284

Herbanios: US; USM!

Nombre común: Desconocido.

Registro departamental: AN, HV, LI.

Regiones Ecológicas: MA, PSH; 3300$3900 \mathrm{~m}$.

SINANPE: PNH

H erbarios peruanos: MOL (2), USM (isotipo+3).

Observaciones: Esta gramínea perenne se conoce de varias localidades, en el centro del país. Asociada a matorrales donde, al parecer, es localmente escasa. Amenazas potenciales a sus poblaciones podrían estar asociadas a los incendios intencionales y modificación del hábitat. 


\section{Festuca lasiorrhachis Pilg.}

\section{DD}

Publicación: Bot. Jahrb. Syst. 37: 508. 1906.

Colección tipo: A. Weberbauer 904; A. Weberbauer $907 \&$ A. Weberbauer 589

Herbarios: B, US.

Nombre común: D esconocido.

Registro departamental: AY, CU, LI, PU. Regiones Ecológicas: PSH; 3700-4100 m.

SINAN PE: RNPG

Herbarios peruanos: CUZ (1).

Observaciones: Esta hierba perenne fue reconocida por Tovar (1993) del Perú y Bolivia. Sin embargo, Soreng et al. (2003) la reconocen como restringida solamente al Perú, pero con problemas taxonómicos, por lo que aquí se la incluye provisionalmente.

\section{Festuca longigluma Tovar}

\section{CR, Bla}

Publicación: Mem. Mus. Hist. Nat. «Javier Prado» 16: 27, t. 5B. 1972.

Colección tipo: R. Ferreyra 6688

Herbarios: US.

Nombre común: D esconocido.

Registro departamental: HU.

Regiones Ecológicas: MA; 3000-3100

$\mathrm{m}$.

SINAN PE: Sin registro.

Hembarios peruanos: Ninguno.

Observaciones: Esta hierba perenne se conoce solamente de una localidad, en el centro del pais, la misma que ha sido herborizada desde fines del siglo XVIII. Sin embargo, esta especie no ha vuelto a ser recolectada desde 1950. La localidad original no recibe protección oficial, y las actividades agro-pastoriles podrían ser amenazas a las poblaciones de esta especie.

\section{Festuca longivaginata Tovar}

\section{EN, B 1ab(iii)}

Publicación: Mem. Mus. Hist. Nat. «Javier Prado» 16: 34, t.7A. 1972.

Colección tipo: E. Cerrate 2474

Herbarios: USM.

Nombre común: D esconocido.

Registro departamental: AN.

Regiones Ecológicas: MA; 2700-3100

m.

SINANPE: Sin registro.

Henbarios peruanos: USM (3).

Observaciones: Esta gramínea perenne se conoce solamente de la cuenca alta del Pativilca, donde, al parecer, no ha vuelto a ser recolectada desde 1956. Habita matorrales subxéricos, los que son poco herborizados en el país y por ello su poca representación en los herbarios. Amenazas a las poblaciones, en este tipo de hábitats, podrían estar asociados por un lado a los incendios intencionales y por el otro a las sequías que afectan continuamente la zona y que incrementan cambios en el paisaje.

\section{Festuca meyenii (St.-Yves) E.B. Alexeev}

$$
\text { EN, Bla }
$$

Publicación: Bot. Zhurn. (Moscow \& Leningrad) 69: 348. 1984.

Colección tipo: G. Meyen s.n.

Herbarios: $B$.

Nombre común: D esconocido.

Registro departamental: AR.

Regiones Ecológicas: PSH; 3900-4200

$\mathrm{m}$.

SINAN PE: Sin registro.

Herbarios peruanos: Ninguno.

Observaciones: Esta gramínea se conoce de dos localidades, solamente de la puna del sur del país. Erróneamente Brako \& Zarucchi (1993) citaron Junín en el rango de distribución de esta especie. D arbyshire etal. (2003) aceptan tentativamente esta especie. Esta especie no ha vuelto, al parecer, a ser recolectada desde 1914, que confirma a Arequipa como una zona de vacío de información botánica. Probablemente podría extenderse a la Reserva Nacional Las Salinas y Aguada Blanca.

\section{Festuca nigniflora (H itchc.) Negrito \& Anton EN, B1ab(iii); D2}

Publicación: Novon 9(4): 542, f. 1. 1999. Colección tipo: J.F. Macbride \& W. Featherstone 1135

Herbarios: BAA, F, US

Nombre común: D esconocido.

Registro departamental: JU, PA.

Regiones Ecológicas: AA; 4200-4600 $\mathrm{m}$.

SINAN PE: Sin registro.

Herbarios penuanos: USM (1).

Observaciones: Esta gramínea se conoce solamente del centro del país. Al igual que otras especies endémicas de los altos Andes, no hay registro adicional de poblaciones. Probablemente hayan poblaciones en la Reserva Nacional de Junín. Habita la zona de puna, la cual está sujeta al pastoreo intensivo y a la quema con fines agrícolas. Se le asigna la categoría En Peligro porque sólo se conoce de dos poblaciones.

\section{Festuca penuviana Infantes}

LC

Publicación: Revista Ci. (Lima) 54: 103. 1952.

Colección tipo: J. Infantes 2449

Herbarios: US,

Nombre común: D esconocido.

Registro departamental: AN, HU, HV, JU, PA.

Regiones Ecológicas: PSH, AA; 3900$4740 \mathrm{~m}$.

SINAN PE: Sin registro.

Herbarios penuanos: USM (21).

Observaciones: Esta gramínea perenne y de porte cespitoso, se conoce de varias localidades, principalmente en el centro del país. Reconocida como endémica por Tovar (1993) y Soreng et al. (2003), un ejemplar no confirmado de Bolivia podría cambiar su rango de distribución. Se considera el estatus de conservación como de preocupación menor. 


\section{Festuca pubigluma Tovar}

$$
\text { EN, Bla }
$$

Publicación: Mem. Mus. Hist. Nat. «Javier Prado» 16: 31, t. 6B. 1972.

Colección tipo: E. Cerrate 2346

Herbarios: MO, US.

Nombre común: D esconocido.

Registro departamental: AN, HU.

Regiones Ecológicas: PSH; 3800-4100

$\mathrm{m}$.

SINANPE: Sin registro.

Herbarios peruanos: USM (1).

Observaciones: Esta gramínea perenne, amacollada, se conoce de dos localidades, en el centro del país. Tovar (1993) indicó su rango restringido a la localidad original, pero una recolecta de 1983, parece ampliar el rango de esta especie. Al igual que otras plantas andinas, amenazas potenciales están asociadas a los incendios intencionales y modificación del hábitat.

\section{Festuca rigidifolia Tovar}

\section{LC}

Publicación: Mem. Mus. Hist. Nat. «Javier Prado» 16: 26, t.5A. 1972.

Colección tipo: W. Rauh \& G. Hirsch P1721

Herbarios: US.

Nombre común: D esconocido. Registro departamental: AN, CU, JU, LI, SM.

Regiones Ecológicas: PSH, AA; 3750$4870 \mathrm{~m}$.

SINANPE: PNH, PNRA, SHMP

Herbarios peruanos: USM?

Observaciones: Esta gramínea perenne se conoce del norte y centro del país. No fue incluida por Brako \& Zarucchi (1993) como endémica, pero sí por Tovar (1993) y Soreng et al. (2003), aunque estos últimos tienen reservas en el estatus taxonómico. Por su área de presencia y representación en tres áreas protegidas se la reconoce como de preocupación menor.

\section{Festuca tarmensis Pilg.}

\section{DD}

Publicación: Bot. Jahrb. Syst. 37: 515516. 1906.

Colección tipo: A. Weberbauer 2233

Herbarios: B, BAA, US; MOL!

Nombre común: D esconocido. Registro departamental: AN, HU, JU, LL, SM.

Regiones Ecológicas: MA, BPM; 3500— $3800 \mathrm{~m}$.

SINANPE: PNRA

Herbarios peruanos: MOL (isotipo).

Observaciones: Esta especie perenne se conoce de varias localidades, en el norte y centro del país, en las cuencas del Palca, Santa y Pativilca. Tovar (1993) la reconoció en un sentido más amplio que el de Soreng et al. (2003). Habrá que aclarar su estatus taxonómico y con ello su rango de distribución en una futura evaluación.

\section{Jarava macbridei (H itchc.) Peñailillo}

\section{LC}

Publicación: Gayana Bot. 59(1): 31. 2002. Colección tipo: J.F. Macbride \& W. Featherstone 452

Hemarios: F, US.

Nombre común: D esconocido.

Registro departamental: AN, CA, HU, LI. Regiones Ecológicas: MDE, MA, PSH, AA; $1400-4600 \mathrm{~m}$.

SINAN PE: Sin registro.

Herbarios peruanos: CPUN (2), USM (7).

Observaciones: Esta gramínea perenne se conoce de varias localidades, en ambientes semixéricos, a lo largo de un amplio rango altitudinal de Cajamarca a Lima. Localmente es frecuente.

\section{Jarava pachypus (Pilg.) Peñailillo}

\section{VU, B lab(iii)}

Publicación: Gayana Bot. 59(1): 32. 2002.

Colección tipo: A. Weberbauer 1487

Herbarios: B, US.

Nombre común: Desconocido.

Registro departamental: AR, IC

Regiones Ecológicas: D ST; $20-620 \mathrm{~m}$.

SINAN PE: Sin registro.

Herbarios peruanos: CPUN (1), USM (2).

Observaciones: Esta gramínea es probablemente una planta anual, si bien Tovar (1993) la considera perenne. Se conoce de cinco localidades, en el sur del país, una de ellas en la Isla de San G allán, en donde fue recolectada en 1919. Todas las poblaciones conocidas están naturalmente fragmentadas. Tovar (1993) comentó el vínculo taxonómico con una especie andina, $\mathrm{N}$ assella nardoides. La colección botánica más reciente proviene de un año de El Niño. Amenazas a algunas de sus poblaciones, probablemente, estén asociadas a modificación de hábitat por expansión urbana.

\section{Merostachys brevispica Munro}

\section{CR, Bla}

Publicación: Trans. Linn. Soc. London 26(1): 49. 1868.

Colección tipo: R. Spruce s.n.

Herbarios: K, US.

Nombre común: Desconocido.

Registro departamental: SM.

Regiones Ecológicas: BMHP; altitud desconocida.

SINANPE: Sin registro.

Herbarios peruanos: Ninguno.

Observaciones: Bambú suberguido, conocido solamente de una localidad, en la cuenca del Mayo. No ha vuelto a ser recolectada desde el siglo XIX. 
49. Muhlenbergia caxamarcensisLaegaard \& Sánchez Vega

$$
\text { VU, B 1ab(iii); D2 }
$$

Publicación: Nordic J. Bot. 10: 437-441. 1990.

Colección tipo: S. Renvoize \& S. Laegaard 4962

Hembarios: AAU, K, MO, US; CPUN!.

Nombre común: D esconocido.

Registro departamental: CA.

Regiones Ecológicas: PAR; 3100-3900 m.

SINAN PE: Sin registro.

Herbarios peruanos: CPUN

(holotipo+7), USM (3).

Observaciones: Esta gramínea se conoce solamente de Cajamarca, donde habita suelos calizos. A fín a M uhlenbergia fastigiata y M . ligularis. Las poblaciones de esta especie son pequeñas y muy cercanas entre sí. En forma similar a otras plantas de lajalca, las poblaciones de esta especie están sujetas a los incendios intencionales y otras actividades humanas que deterioran el hábitat de éstas.

50. Muhlenbergia flexuosa $\mathrm{H}$ itchc.

$$
\text { VU, B lab(iii) }
$$

Publicación: Contr. U.S. Natl. Herb. 24(8): 388. 1927.

Colección tipo: J.F. Macbride 3874

Herbarios: F, LE, US.

Nombre común: D esconocido.

Registro departamental: $\mathrm{HU}, \mathrm{PA}$

Regiones Ecológicas: BMHM, BMHP; 1200- $2000 \mathrm{~m}$.

SINAN PE: PNYC

Herbarios peruanos: USM (2).

Observaciones: Esta gramínea perenne se conoce solamente de tres localidades, en el centro del país, incluyendo una en el Parque Nacional Yanachaga-Chemillén. Localmente es una planta rara. Como una típica gramínea $\mathrm{C}$, habita ambientes abiertos y expuestos a extremos de temperatura.

\section{Muhlenbergia maxima Laegaand \& Sánchez Vega EN, Blab(iii)}

Publicación: Nordic J. Bot. 10: 439. 1990. Colección tipo: I. Sánchez V. \& W. Ruiz V. 3561

Herbarios: AAU, K, US; CPUN!, USM!.

Nombre común: D esconocido.

Registro departamental: AM, CA.

Regiones Ecológicas: MA; 2100-3100 m.

SINANPE: Sin registro.

Herbarios penuanos: CPUN (holotipo+2), USM (isotipo).

Observaciones: Esta especie se conoce de pocas localidades, en el norte del país, en la cuenca del río Marañón. Amenazas a las poblaciones proviene de modificación del hábitat por programas de reforestación con eucalipto.

\section{Muhlenbergia monandra Alegría \& Rúgolo}

EN, Blab(iii); D2

Publicación: Darwiniana 39(1-2): 20, f. 1-3.. 2001.

Colección tipo: A. Granda \& J.J. Alegría 2230

Herbarios: SI, US; MOL!, USM!

Nombre común: D esconocido.

Registro departamental: LI.

Regiones Ecológicas: MA; 2700-2800 m. SINANPE: Sin registro.

Herbarios penuanos: MOL (holotipo+1), USM (isotipo).

Observaciones: Esta es una especie anual conocida solamente de dos localidades, ambas en el valle del Chillón. Una de las localidades está a unos $12 \mathrm{~km}$ de distancia, cerca de Puruchuco. Tal vez su caracter anual ha permitido que pase desapercibida.

\section{Nassella ayacuchensis (Tovar) Barkworth}

$$
\text { CR, Bla }
$$

Publicación: Taxon 39(4): 609. 1990.

Colección tipo: O. Tovar 5492

Herbarios: US; USM!

Nombre común: D esconocido.

Registro departamental: AY.

Regiones Ecológicas: MDE, MA; 1800- $2900 \mathrm{~m}$.

SINAN PE: Sin registro.

Herbarios peruanos: USM (isotipo).

Observaciones: Esta especie proviene de uno delos departamentos con menor cantidad de colecciones en los herbarios. Solamente se conoce de la localidad original herborizada en 1966.

\section{Nassella huallancaensis (Tovar) Barkworth}

$$
\text { CR, Bla }
$$

Publicación: Taxon 39(4): 610. 1990.

Colección tipo: O. Tovar \& S. RivasMartinez 9880

Herbarios: MAF, MO, US; USM!.

Nombre común: D esconocido.

Registro departamental: HU.

Regiones Ecológicas: PSH; 3700 m.

SINAN PE: Sin registro.

Herbarios peruanos: USM (isotipo).

Observaciones: Esta especie perenne se conoce solamente de una localidad en la cuenca alta del Marañón. Esta localidad ha sido escasamente explorada para este tipo de plantas. No se conoce de amenazas, ni el tamaño de las poblaciones. Por su área de presencia restringida se la reconoce como amenazada.

\section{Nassella soukupii (Tovar) Barkworth}

$$
\text { CR, Bla }
$$

Publicación: Taxon 39(4): 612. 1990.

Colección tipo: O. Tovar 2814

Hemanios: MO, US; USM!

Nombre común: D esconocido.

Registro departamental: JU.

Regiones Ecológicas: PSH; $3800 \mathrm{~m}$.

SINANPE: Sin registro.

Herbarios peruanos: USM (holotipo). 
Observaciones: Esta hierba cespitosa y perenne se conoce solamente de una localidad, en el centro del país, en una de las zonas más intensamente empleadas en actividades agro-pastoriles. No ha vuelto a ser recolectada desde 1957.

\section{Nassella vargasii (Tovar) Peñailillo$$
\text { CR, Bla }
$$

Publicación: Gayana, Bot. 55(2): 87. [1999] 1998.

Colección tipo: C. Vargas C. 14127

Herbarios: US.

Nombre común: D esconocido.

Registro departamental: CU.

Regiones Ecológicas: MA; 2860-3000 m.

SINAN PE: Sin registro.

Herbarios peruanos: USM (1).

Observaciones: Esta planta cespitosa y perenne se conoce de una localidad, en la cuenca del río Urubamba. No ha vuelto a ser recolectada desde 1966. Se carece de información acerca del estado actual de su población. Por su área de presencia restringida se la reconoce como amenazada.

\section{Nassella wurdackii (Tovar) Barkworth}

\section{EN, Bla}

Publicación: Taxon 39(4): 612. 1990.

Colección tipo: J.J. Wurdack 1152

Herbarios: US.

Nombre común: Desconocido.

Registro departamental: AM, CU.

Regiones Ecológicas: PAR, BPM; 3100$3500 \mathrm{~m}$.

SINANPE: Sin registro.

Herbarios peruanos: Ninguno.

Observaciones: Esta especie cespitosa y perenne se conoce solamente de dos localidades, distantes entre ellas por más de 500 $\mathrm{km}$ en línea recta. Probablemente, otras poblaciones se encuentren en los pajonales ecotonales con la parte alta del bosque montano.

\section{Paspalum anderssonii Mez}

\section{DD}

Publicación: Repert. Spec. Nov. Regni Veg. 15: 71.1917.

Colección tipo: N.J. Andersson s.n.

Hembarios: B, US.

Nombre común: D esconocido.

Registro departamental: Sin datos.

Regiones Ecológicas: Sin datos.

SINAN PE: Sin registro.

Herbarios peruanos: Ninguno.

Observaciones: Esta hierba perenne se conoce de la colección tipo, una planta recolectada a mediados del siglo XIX, de procedencia desconocida. A parentementeno havuelto a ser recolectadani confimada para el país, si bien se la reconoce como una buena entidad biológica.

\section{Paspalum barclayi Chase}

\section{VU, Bla}

Publicación: Contr. U.S. Natl. Herb. 24(8): 452. 1927.

Colección tipo: G.W. Barclay 311

Herbarios: MO, US.

Nombre común: D esconocido.

Registro departamental: AM, LI.

Regiones Ecológicas: D ST, MDE; 0$2300 \mathrm{~m}$.

SINANPE: Sin registro.
Henbarios peruanos: USM (1).

Observaciones: Esta gramínea perenne se conoce según Tovar (1993) de dos localidades, disyuntas por más de $500 \mathrm{~km}$ de distancia. La localidad oniginal se halla en los alrededores deCallao, unadelas provincias más densamente urbanizadas en la costa. Por tratarse de una planta con fotosíntesis C4 podría estar más extendida en sitios abiertos, los que en general son poco herborizados. Amenazas potenciales incluyen, para el caso de poblaciones en la costa, la expansión urbana.

\section{Paspalum haenkeanum J. Presl}

\section{LC}

Publicación: Reliq. Haenk. 1(4- 5): 210. 1830.

Colección tipo: T. Haenke s.n.

Herbarios: BM, MO, PR.

Nombre común: Grama, nudillo.

Registro departamental: CA, HU, IC, LI, LL, TU.

Regiones Ecológicas: DCT, D ST; 25$500 \mathrm{~m}$.

SINANPE: Sin registro.

Herbarios penuanos: CPUN (2), HUT (2), USM (3).

Observaciones: Esta gramínea perenne se conoce de varias localidades, ubicadas en la llanura costera. Sagástegui \& Leiva (1993) la incluyeron como maleza de campos de cultivo, pero en realidad esta endémica con fotosíntesis C4 es capaz de habitar en ambientes abiertos y modificados.

\section{Paspalum killipii (Hitchc.) Zuloaga \& Soderstr.}

$$
\text { EN, Bla }
$$

Publicación: Smithsonian Contr. Bot. 59: 40. 1985.

Colección tipo: E.P. Killip \& A.C. Smith 22804

Herbarios: US.

Nombre común: D esconocido.

Registro departamental: $\mathrm{AY}, \mathrm{CU}, \mathrm{HV}$.

Regiones Ecológicas: BMHP; 750- $1200 \mathrm{~m}$.

SINANPE: Sin registro.

Herbarios penuanos: USM (1).

Observaciones: Esta gramínea perenne se conoce de tres localidades en el sur del país, de las cuencas de los ríos Apurímac y Mantaro, localidades con escasa información botánica reciente. Esta especie aparentemente no ha vuelto a ser recolectada desde 1964.

\section{Paspalum soukupii Carbonó}

\section{CR, Blab(iii)}

Publicación: Ann. Missouri Bot. Gard. 82(1): 112-113, f. 15. 1995.

Colección tipo: J. Soukup 5282

Henbarios: US.

Nombre común: Desconocido.

Registro departamental: LI.

Regiones Ecológicas: MDE; 2000- 2600 m.

SINAN PE: Sin registro.

Herbarios peruanos: Ninguno.

Observaciones: Gramínea anual conocida de dos localidades cercanas entre ellas en la cuenca del río Rímac. Esta especie tiene vínculos cercanos con Paspalum candidum, y por ello el ejemplar original fue reconocido por Tovar (1993) como un taxón en la sinonimia de esa especie. Aparentemente no ha vuelto a ser recolectada desde 1964. Esta especie crece en ambientes rocosos, los que han sido escasamente recolectados. Sin embargo, la cuenca del Rímac es una de las más modificadas por la acción humana, por lo que se la considera en peligro crítico. 


\section{Paspalum tuberosum Mez}

\section{LC}

Publicación: Repert. Spec. Nov. Regni Veg. 15: 29. 1917.

Colección tipo: A. Weberbauer 3815; A. Weberbauer 3932

Hemarios: B, US

Nombre común: D esconocido.

Registro departamental: AN, CA, LL.

Regiones Ecológicas: MA, PSH; 2400$3900 \mathrm{~m}$.

SINANPE: PNH

Herbarios penuanos: CPUN (2), USM (2).

Observaciones: G ramínea conocida de varias localidades dispersas, principalmente en la vertiente del Pacífico. Fue descrita de plantas recolectadas entre las cuencas del Saña y Magdalena. Se conoce también de la cuenca del Santa.

\section{Pennisetum annuum Mez}

\section{LC}

Publicación: Bot. Jahrb. Syst. 56(Beibl. 125): 7. 1921.

Colección tipo: A. Weberbauer 5354

Herbarios: B, F.

Nombre común: D esconocido.

Registro departamental: CA, LL, LI.

Regiones Ecológicas: DST, MDE; 650 $2000 \mathrm{~m}$.

SINANPE: Sin registro.

Herbarios peruanos: USM (4).

Observaciones: Estaesunaespecieanual, conocida devarias localidades dispersas, a lo largo de la costa y piedemonte occidental andino. O cupa ambientes abiertos en las estribaciones andinasyal igual que otras plantas de ambientes disturbados es poco el registro botánico.

\section{Pennisetum nupestre Chase}

\section{LC}

Publicación: Contr. U.S. Natl. Herb. 24(8): 484. 1927.

Colección tipo: J.F. Macbride \& W. Featherstone 453

Hemarios: F, US.

Nombre común: D esconocido.

Registro departamental: $\mathrm{AN}, \mathrm{AY}, \mathrm{CA}$, HV, JU, LI.

Regiones Ecológicas: MDE, MA; 2000$3000 \mathrm{~m}$.

SINAN PE: Sin registro.

Herbarios peruanos: USM (38).

Observaciones: Esta especie perenne se conoce de varias localidades, en los valles interandinos y vertientes occidentales. Se conoce de ambientes semixéricos.

\section{Piptochaetium featherstonei (Hitchc.) Tovar}

\section{NT}

Publicación: O pusc. Bot. Pharm. Complut. 4: 104. 1988.

Colección tipo: J.F. Macbride \& W. Featherstone 803A

Herbarios: F, US.

Nombre común: D esconocido.

Registro departamental: AN, AY, JU, LI. Regiones Ecológicas: PSH, AA; 3500$4500 \mathrm{~m}$.
SINAN PE: Sin registro.

Herbarios peruanos: CPUN (3), USM (6).

Observaciones: Hierba perenney cespitosa conocida de pajonales, en el centro y sur del país. Amenazas a sus poblaciones probablemente, sean de carácter local y relacionadas con los incendios intencionales, pastoreo intensivo y cambio de hábitat por la minería.

\section{Piptochaetium sagasteguii Sánchez Vega CR, Blab(iii)}

Publicación: Arnaldoa 1(1): 17. 1991. Colección tipo: I. Sánchez V. 2914 Hemarios: AAU, F, K, MO, US; CPUN!, HAO!, USM!

Nombre común: D esconocido.

Registro departamental: CA.

Regiones Ecológicas: MA; 2800 m.

SINANPE: Sin registro.

Herbarios peruanos: CPUN (holotipo), HAO (isotipo), USM (isotipo).

Observaciones: Hierba perenne conocida solamente de los alrededores del valle de Cajamarca. Amenazas a su población está relacionada con la minería.

\section{Piptochaetium tovanii Sánchez Vega subsp. pilosum Sánchez Vega}

EN, B1ab(iii)

Publicación: Arnaldoa 1(1): 29. 1991.

Colección tipo: I. Sánchez V. et al. 2459

Herbarios: AAU, CHAPA, F, K, MO; CPUN, HUT, USM!.

Nombre común: D esconocido.

Registro departamental: CA, JU, PI.

Regiones Ecológicas: MA, PAR; 2720$3800 \mathrm{~m}$.

SINAN PE: Sin registro.

Herbarios peruanos: CPUN (holotipo+5), HUT (isotipo), USM (isotipo).

Observaciones: Hierba perenne conocida solamente de dos localidades. Sin embargo, la localidad original ha sido destruida por reforestación con eucalipto.

\section{Poa anae Tovar}

\section{EN, Bla}

Publicación: Publ. Mus. Hist. Nat. «Javier Prado», Ser. B, Bot. 33: 6. 1985.

Colección tipo: O. Tovar 9257

Herbarios: MAF, MO, US; USM!.

Nombre común: D esconocido.

Registro departamental: CA, JU, LI.

Regiones Ecológicas: PAR, AA; 4050$5500 \mathrm{~m}$.

SINAN PE: Sin registro.

Herbarios peruanos: CPUN (2), USM (isotipo+2).

Observaciones: Esta especie cespitosa y perenne se conoce de localidades altoandinas, por encima de $\operatorname{los} 4000 \mathrm{~m}$. Estas localidades se hallan en forma disyunta de Cajamarca al centro del país; probablemente existan otras poblaciones en otros departamentos. Amenazas potenciales a sus poblaciones probablemente estén asociadas a la modificación de hábitat por la actividad minera, especialmente durante estos últimos 20 años con el retroceso de glaciares. 


\section{Poa ayacuchensis Tovar}

\section{CR, Bla}

Publicación: Bol. Soc. Penuana Bot. 7: 6. 1974. Colección tipo: O. Tovar \& Foguel 7007

Herbarios: MO, US; USM!.

Nombre común: D esconocido.

Registro departamental: AY.

Regiones Ecológicas: PSH; $3700 \mathrm{~m}$.

SINAN PE: Sin registro.

Herbarios peruanos: USM (holotipo).

Observaciones: Esta hierba perenne se conoce solamente de una localidad, la cual está ubicada en la vertiente occidental de Ayacucho, uno de los departamentos con vacío de información botánica, especialmente en las zonas altoandinas.

\section{Poa carazensis Pilg.}

\section{EN, Blab(iii)}

Publicación: Bot. Jahrb. Syst. 37: 380381. 1906.

Colección tipo: A. Weberbauer 3073

Hemarios: BAA, MO, US; MOL!.

Nombre común: D esconocido.

Registro departamental: AN, CA.

Regiones Ecológicas: MA, $\mathrm{PSH}, \mathrm{AA}$; 2700- $4500 \mathrm{~m}$.

SINANPE: PNH

Herbarios peruanos: HUT (1), MOL (isotipo), USM?.

Observaciones: Planta cespitosa y perenne conocida de menos de cinco localidades. Si bien está representada en el Parque Nacional Huascarán, sus poblaciones pueden estar afectadas por incendios intencionales y modificación del hábitat.

\section{Poa ferreyrae Tovar}

\section{DD}

Publicación: Mem. Mus. Hist. Nat. «Javier Prado» 15: 37, t.8B. 1965.

Colección tipo: R. Ferreyra 7619

Herbarios: MO, US; MOL!

Nombre común: Desconocido.

Registro departamental: CA, LL.

Regiones Ecológicas: MA, PAR; 3000$3650 \mathrm{~m}$.

SINAN PE: Sin registro.

Herbarios peruanos: CPUN (1), MOL (isotipo), USM (1).

Observaciones: Planta perenne, conocida de tres localidades aisladas en el norte del país, todas ubicadas en la vertiente occidental. Especie vinculada a Poa fibrifera, especie del Perú y Bolivia y a Poa tovarii con las que parece ser coespecífica.

\section{Poa huancavelicae Tovar}

\section{EN, Bla}

Publicación: Mem. Mus. Hist. Nat. «Javier Prado» 15: 52, t.12A. 1965.

Colección tipo: O. Tovar 2846

Herbarios: MO ; USM!.

Nombre común: D esconocido.

Registro departamental: CU, HV.

Regiones Ecológicas: PSH, AA; 4065$4600 \mathrm{~m}$.

SINAN PE: Sin registro
Herbarios peruanos: USM (holotipo).

Observaciones: Esta hierba perenne fue descrita de una planta recolectada en la cuenca del Pampas, en 1958. Una población adicional fue registrada en 1971, a unos $350 \mathrm{~km}$ de distancia en una localidad no precisada entre el Abra Málaga y Pisac (Cusco). Soreng et al. (2003) han aceptado tentativamente esta especie, por lo que estudios taxonómicos futuros podrían aclarar su vínculo con otras especies andinas.

\section{Poa marshallii Tovar}

\section{EN, Bla}

Publicación: Revista Ci. U.N.M.S.M. 73(1): 103. 1981.

Colección tipo: J.R. Lloyd \& J.K. Marshall 241

Herbarios: K, MO.

Nombre común: Desconocido.

Registro departamental: $\mathrm{AY}, \mathrm{HV}$, JU.

Regiones Ecológicas: PSH, AA; 4000$4700 \mathrm{~m}$.

SINAN PE: Sin registro.

Herbarios peruanos: USM (6).

Observaciones: Hierba perenne,conocida del centro del país. La localidad original erróneamente se atribuyó a Junín, pero Acobambilla está localizada en Huancavelica, en una subcuenca del Mantaro. Esta especie forma parte de un complejo de especies con límites poco claros, por lo que Soreng et al. (2003) la reconocen tentativamente. Se espera que estudios futuros aclaren la taxonomía de esta especie. Por su área de presencia menor a $5000 \mathrm{~km}^{2}$ se considera una especie en peligro.

\section{Poa oscariana N egritto \& Anton}

\section{EN, Bla}

Publicación: Syst. Bot. 31(1): 84, 88, f. 2. 2006.

Colección tipo: A. Cano 3802

Henbarios: ; USM!.

Nombre común: D esconocido.

Registro departamental: CU.

Regiones Ecológicas: PSH, BPM; 3600$4030 \mathrm{~m}$.

SINANPE: PNM

Herbarios penuanos: USM (holotipo+1).

Observaciones: Hierba perenne, conocida del sur oriente del país, donde habita ambientes ecotonales bosque-pajonal. Fue descrita de una planta recolectada en 1990, del límite occidental del Parque Nacional Manu.

\section{Poa pilgeri N egritto \& Anton}

$$
\text { EN, Bla }
$$

Publicación: Syst. Bot. 31(1): 88, f. 3. 2006. Colección tipo: I.J. Blair 424

Hemarios: $\mathrm{K}$.

Nombre común: Desconocido.

Registro departamental: JU.

Regiones Ecológicas: PSH; $3900 \mathrm{~m}$.

SINAN PE: Sin registro.

Herbarios peruanos: Ninguno.

Observaciones: Hierba perenne, descrita de una colección realizada en un pajonal disturbado, por pastoreo intensivo. La especie ha sido descrita como ginomonoica, con flores basales perfectas. 


\section{Poa scabrivaginata Tovar}

$$
\text { VU, B lab(iii) }
$$

Publicación: Mem. Mus. Hist. Nat. «Javier Prado» 15: 48, t.11A. 1965.

Colección tipo: J.F. Macbride 4354

Herbarios: K, US.

Nombre común: D esconocido.

Registro departamental: AN, HU, HV.

Regiones Ecológicas: PSH, AA; 3962$4100 \mathrm{~m}$.

SINANPE: Sin registro.

Herbarios peruanos: USM (1).

Obsenvaciones: Esta hierba perenne se conoce del centro del país, de localidades aisladas. Soreng et al. (2003) reconocen tentativamente a estaespecie, puesformapartedeun complejo queincluyeaPoa aequatoriensis deEcuadory Perú. Habitala zona de puna, la cual está sujetaal pastoreo intensivo y a la quema con fines agnícolas y pecuarios.

\section{Poa tovanii Soreng}

\section{DD}

Publicación: Novon 8(2): 200. «ovari» 1998. Colección tipo: E. Anderson 1265

Herbarios: MO, US.

Nombre común: D esconocido.

Registro departamental: JU, LL, TA.

Regiones Ecológicas: MA, PSH; 3100$4060 \mathrm{~m}$.

SINAN PE: Sin registro.

Herbarios peruanos: USM (1).

Observaciones: Esta hierba perenne se conoce de tres localidades aisladas y dispersas, a lo largo de los Andes peruanos, de La Libertad a Tacna. La localidad original es la misma que de otra especie endémica Poa ferregrae.

\section{Setaria condobensis R.A.W. Herrm. var. limense (Tovar) Pensiero}

\section{EN, B 1ab(iii)}

Publicación: Darwiniana 37(1-2): 56, f. 28. 1999.

Colección tipo: R. Ferreyra 9154

Herbarios: MO, US.

Nombre común: D esconocido.

Registro departamental: LI.

Regiones Ecológicas: MDE; 1700 - 2000 m.

SINANPE: Sin registro.

Herbarios peruanos: USM (1).

Observaciones: Esta gramínea bianual se conoce solamente de la vertiente occidental y de la cuenca media del Rímac. Esta especie habita ambientes expuestos a temperaturas extremas. Amenazas potenciales están asociadas a cambios en el hábitat por expansión de caminos y lugares urbanos.

\section{Sponobolus mirabilis Pilg.}

\section{CR, Blab(iii)}

Publicación: Bot. Jahrb. Syst. 56(Beibl. 123): 27. 1920

Colección tipo: A. Weberbauer 6186

Herbarios: B, BAA.

Nombre común: Desconocido.

Registro departamental: $\mathrm{CA}$.

Regiones Ecológicas: BS, BMHP; 700$800 \mathrm{~m}$.

SINANPE: Sin registro.

Herbarios peruanos: Ninguno.

Observaciones: Esta gramínea perenne se conoce solamente de una localidad, ubicada en un tributario del Chinchipe. No ha vuelto a ser recolectada desde 1912, al parecer crecía en un sotobosque. Es una especie asociada a bosques, amenazas a sus poblaciones están asociadas a la deforestación, en uno de los departamentos de mayor incremento poblacional.

\section{Uniola peruviana Laegaand \& Sánchez Vega}

\section{EN, Blab(iii)}

Publicación: Nordic J. Bot. 10(4): 439. 1990.

Colección tipo: I. Sánchez V. 2642

Hemarios: AAU, K; CPUN!.

Nombre común: D esconocido.

Registro departamental: AM.

Regiones Ecológicas: BS; 430-1300 m. SINANPE: Sin registro.

Herbarios penuanos: CPUN (holotipo+1), HAO (1), HUT (1).

Observaciones: Hierba conocida solamente de la cuenca del río Utcubamba. La localidad original está muy modificada por tala y pastoreo intensivo. Por su distribución restringida y amenazas conocidas a una de las subpoblaciones se la reconoce amenazada. 
Mapa del Perú indicando las abreviaturas de los departamentos

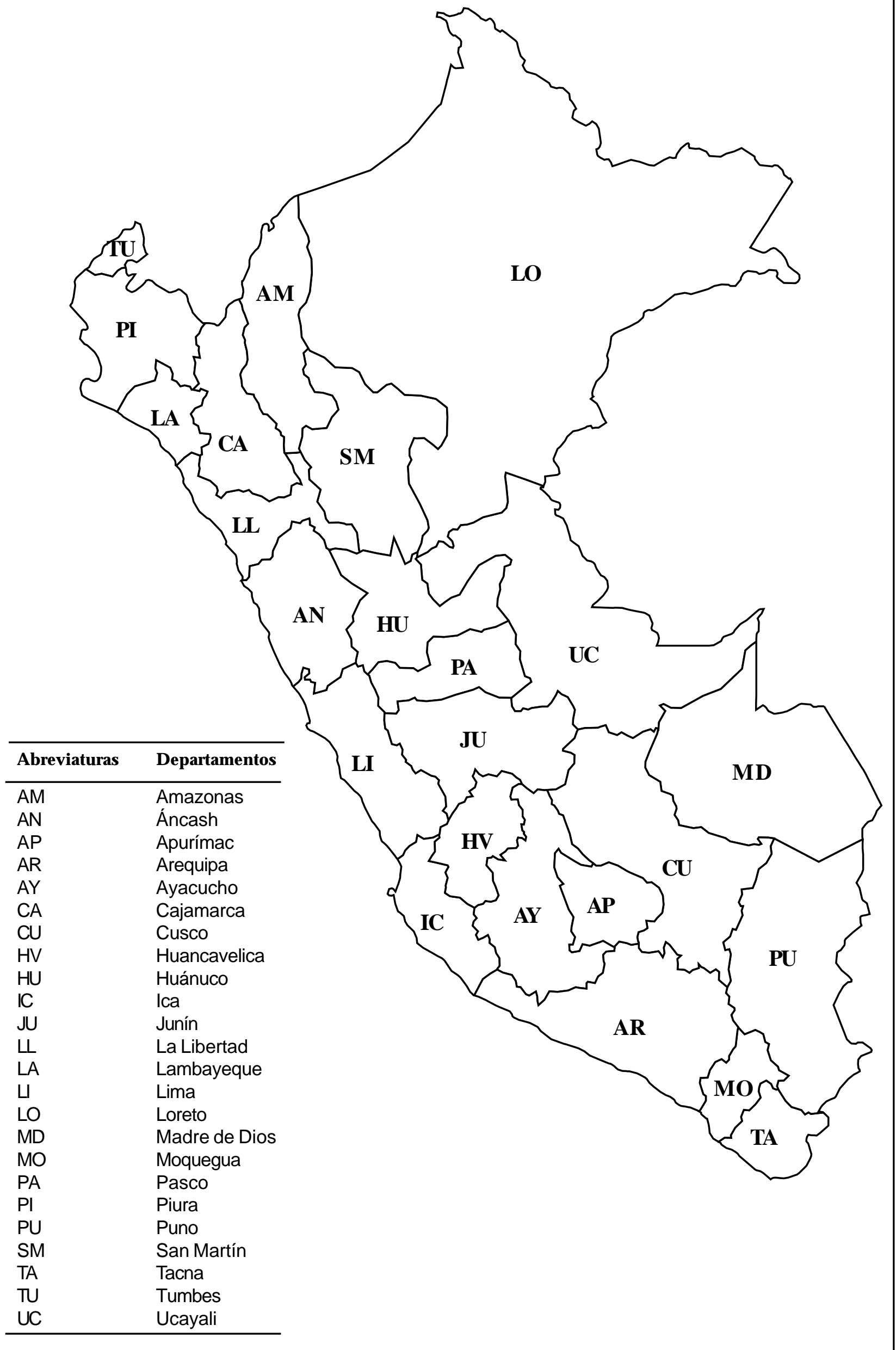

